\title{
Inheriting the benefits of enrichment
}

It is never too early to start enriching a child's mind, some claim, which is why many pregnant women read aloud to their unborn babies or expose them to classical music. A new study in mice suggests that truly diligent mothers-to-be can begin such efforts even earlier-before their children are even conceived.

Environmental enrichment is known to benefit brain function in animals, and previous research led by Larry Feig (Tufts University School of Medicine, Boston, MA) found that young mice that were exposed to enrichment showed improvements in long-term potentiation, a synaptic mechanism associated with learning and memory. Now, Feig and colleagues have discovered that enriched female mice can pass these benefits on to their future offspring (J. Neurosci. 29, 1496-1502; 2009).

The researchers examined wild-type mice and mutant mice with genetically engineered defects in long-term potentiation and in memory formation. For several hours a day for two weeks,

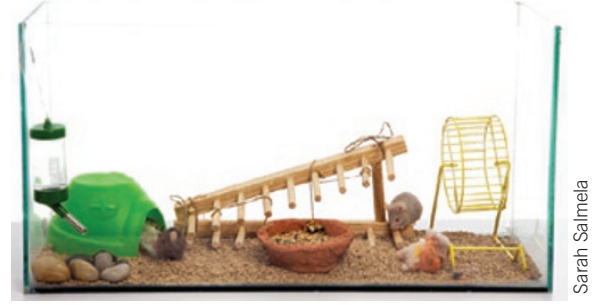

impaired, they performed better in a memory acquisition task than did mutant mice born to nonenriched dams. The benefits were transient, disappearing by the time mice were about 3 months old.

The effects of enrichment were passed on by the dam only (it did not matter whether the sire had been exposed to enrichment) and were transmitted to only one generation. The effects were not a result of nurturing: when biological offspring of enriched dams were raised by nonenriched foster females, the effects were still observed, whereas pups born to nonenriched mothers but raised by enriched foster females did not show enhanced long-term potentiation or memory acquisition.

This research suggests that exposure to environmental enrichment can create heritable traits that are not reflected in changes to DNA structure. Feig and colleagues speculate that the source of the effect may be hormonal and are currently working to uncover the mechanisms underlying it.

Karen Marron

\section{HOW MOSOUTTOOES EIGHT MALARIA}

Malaria infects as many half a billion people every year and kills between 1 and 3 million people annually, many of them children living in sub-Saharan Africa. It is caused by Plasmodium spp. protozoans transmitted between humans and mosquitoes via the blood. When a mosquito consumes infected human blood, its immune system attacks Plasmodium and kills roughly $80-90 \%$ of the parasites, but the surviving parasites multiply and are then transmitted to other humans by mosquito bites, spreading the disease. A recent study has elucidated the mechanism by which the mosquito's immune system responds to Plasmodium. The results raise the possibility that we could learn to enhance this immune response, enabling mosquitoes to eliminate Plasmodium more effectively and minimizing malaria transmission from mosquitoes to humans.

The study, led by George K. Christophides and colleagues (Imperial College London, UK), focused on Anopheles gambiae mosquitoes infected with Plasmodium berghei, a species that causes malaria in rodents but not in humans. Christophides' group identified two proteins, called LRIM1 and APL1C, that seem to mediate the mosquitoes' immune attack on $P$. berghei (Science, published online 5 March 2009; doi:10.1126/science.1171400). LRIM1 and APL1C form a complex that is secreted into the hemolymph, where it interacts with a third protein called TEP1. The LRIM1-APL1C complex may process TEP1 into an active form or help stabilize it in the hemolymph. TEP1 then binds to $P$. berghei parasites and kills them. The nature of the interaction between TEP1 and the LRIM1-APL1C complex warrants further investigation. More studies are also needed to determine how some Plasmodium parasites escape the mosquitoes' immune response.

LRIM1 and APL1C proteins contain repeating amino acid sequences that are rich in leucine. Such leucine-rich repeat-containing (LRR) proteins are important in host defense in both plants and animals. LRIM1 and APL1C belong to a family of LLR proteins that seems to be specific to mosquitoes; proteins with similar structural features have been found in mosquito vectors of other infectious diseases (such as Aedes egyptii, which can transmit yellow and dengue fevers, and Culex quinquefasciatus, which can transmit filariasis) but not in any other species. Christophides and his fellow researchers are investigating whether mosquitospecific LRR proteins are involved in immune responses to these infections as well. If they are, the results of the future studies could have implications for controlling various dangerous human pathogens. 